BULL. AUSTRAL. MATH. SOC.

$18 \mathrm{B25}, 20 \mathrm{MO} 7,20 \mathrm{MIO}$

VOL. $26(1982), 471-472$.

\title{
SEMIGROUPS IN A TOPOS AND STRUCTURE THEORY \\ IN INVERSE SEMIGROUPS
}

\author{
RosS WILKINSON
}

This thesis is in two parts. In the first part we define a semigroup in a topos and provide constructions that enable the basics of an elementary theory of semigroups to be set out. In the second part we examine a verbal product of certain types of varieties of inverse semigroups and examine certain structures lying within this product.

We present a topos as an abstract category, with no reference to set theory and hom-functors, together with explicit constructions and examples needed to develop a theory of semigroups. By taking a constructive approach we are able to construct semigroups in various topoi. For instance we see that topological semigroups are semigroups in one particular topos. Chapter 2, much of which was done jointly with a fellow student, C.A. Read, develops some of the key elements of a semigroup theory within a topos. We first give some basic definitions and results and then give a series of examples of semigroups naturally residing in any topos as well as examples residing in specific topoi. In particular we construct arrows internalizing composition of arrows and composition of relations. Finally we develop the factor semigroup, important for its fundamental position in semigroup theory, and also in this case for its construction which illustrates many important constructive tools needed within a topos.

In Chapter 3, we introduce the concept of a verbal variety of inverse semigroups, and then show that within this collection of varieties, a verbal product generalizing the one given by Neumann [2] is associative.

Received 13 August 1982. Thesis submitted to Monash University, February 1982. Degree approved August 1982. Supervisor: Professor G.B. Preston. 
For verbal varieties of semilattices of groups, this product is shown to be the same as a product generated by extensions of semilattices of groups in the first variety by semilattices of groups in the second variety. This mirrors a similar result for varieties of groups [2]. We then give a lattice structure to this collection of verbal varieties and find that no verbal variety consists solely of semilattices of groups unless it is the variety of semilattices.

Chapter 4 examines some of the constructions that lie within the verbal product, the most fundamental being the semi-direct product of inverse semigroups. We give some important examples of semi-direct products, including semilattices of groups and an inverse semigroup with the symetric inverse semigroup on a set $X$ as an idempotent separating morphic image and we thus deduce a result of McAlister [1] that every inverse semigroup is an idempotent separating morphic image of an $E$-unitary inverse semigroup. We then give a new characterization of $E$-unitary inverse semigroups in terms of a semi-direct product of a group and a semilattice. Finally we introduce a definition of the wreath product of inverse semigroups and see that this product also lies within the verbal product.

\section{References}

[1] D.B. McAlister, "Groups, semilattices and inverse semigroups", Trans. Amer. Math. Soc. 192 (1974), 227-244.

[2] Hanna Neumann, Varieties of groups (Ergebnisse der Mathematik und ihrer Grenzgebiete, 37. Springer-Verlag, Berlin, Heidelberg, New York, 1967).

Department of Mathematics, Monash University, Clayton, Victoria 3168 , Australia. 